\title{
MICF: A Fusion Propulsion System for Interstellar Missions
}

\author{
Terry Kammash ${ }^{1}$ and Brice N. Cassenti ${ }^{2}$ \\ ${ }^{I}$ Nuclear Engineering and Radiological Sciences Dept., University of Michigan, Ann Arbor, MI 48109 \\ ${ }^{2}$ United Technologies Research Center, 411 Silver Lane, MS 129-73, East Hartford, CT 06108 \\ l(734) 764-0205 (voice), (734) 763-4540 (fax), tkammash@umich.edu \\ 2(860)610-7460(voice), (860)610-7536(fax), cassenbn@utrc.utc.com
}

\begin{abstract}
A very promising propulsion device that could open up the solar system and beyond to human exploration is the Magnetically Insulated Inertial Confinement Fusion (MICF) system. This scheme combines the favorable aspects of inertial and magnetic fusion into one where physical containment of the hot plasma is provided by a metal shell while its thermal energy is insulated from this wall by a strong, self-generated magnetic field. The fusion nuclear reactions in this device can be triggered by a beam of antiprotons that enters the target through a hole and annihilates on the deuterium-tritium (DT) coated inner wall giving rise to the hot fusion plasma. In addition to thermally insulating the plasma, the magnetic field helps to contain the charged annihilation products and allows them to deposit their energy in the plasma to heat it to thermonuclear temperatures. Preliminary analysis given in this paper shows that an MICF propulsion system is capable of producing specific impulses on the order of $10^{6}$ seconds. Such capability makes not only the most distant planet in the solar system, but also the nearest star reachable in a human's lifetime. It also shows that a robotic mission to $10,000 \mathrm{AU}$ can readily be achieved in less than 50 years.
\end{abstract}

\section{INTRODUCTION}

The Magnetically Insulated Inertial Confinement Fusion (MICF) concept (Hasegawa, 1988 and Kammash, 1989) illustrated in Fig. 1 represents a novel approach to fusion in that it combines the desirable confinement features of both magnetic and inertial fusions into one. In this scheme a core of plasma is created at the center of a hollowshell by bombarding the fuel-coated inner surface with a laser or particle beam that enters the shell through a hole. The ablated electrons form current loops (Sawanakamp, 1986) which in turn give rise to a strong magnetic field that serves to thermally insulate the plasma from the solid walls. Although different theories have been advanced to explain the mechanism for the generation of such a field a plausible explanation revolves around the formation of density and temperature gradients in the ablation region. According to the generalized Ohm's law these gradients, when at an angle with respect to one another, give rise to an electric field which in turn gives rise to a time-varying magnetic field.

The major advantage of MICF over conventional "implosion" type inertial fusion is the long lifetime of the plasma which allows it to burn longer and generate more fusion energy as represented by very large gain factors. This lifetime is dictated by the time it takes a shock wave to traverse the metal shell that surrounds the plasma once it is formed when the laser or particle beam strikes the inner wall. The sound speed in the shell is much slower than that in the plasma (which dictates the lifetime in the implosion type system) due to the larger atomic mass of the metal shell and its lower temperature that results from the thermal insulation provided by the self-generated magnetic field. Morcover the beam plasma coupling is much more efficient since the energy is put directly into the plasma rather than in an imploding pusher. The Rayleigh-Taylor instability which is known to plague the implosion type inertial fusion is totally eliminated in MICF due to the fact that the lighter fluid (the plasma) is supported by the heavier fluid (the shell) in the presence of a gravitational field. These unique properties allow for a significantly higher

CP504, Space Technology and Applications International Forum-2000, edited by M. S. El-Genk

(C) 2000 American Institute of Physics 1-56396-919-X/00/\$17.00 
energy multiplication factors making MICF a particularly attractive fusion power. As a propulsion system (Kammash, 1987) it is envisaged that MICF pellets injected into a burn chamber will be triggered by an incident laser or particle beam at the center, and the reaction products along with the ionized shell material exhausted through a magnetic nozzle which will be an integral part of an externally-applied magnetic configuration that also serves to cushion the chamber walls from the micro-explosion shock.

In this paper we will examine the propulsion capability of an anti-proton driven MICF and apply the results to an interstellar mission. Specifically we will address a robotic exploration mission to 10,000 AU and show that it can be done in less than 50 years.

\section{PROPULSION ANALYSIS OF MICF}

We consider a target such as that shown in Fig. 1 with a radius of $1 \mathrm{~cm}$. and a core of $0.25 \mathrm{~cm}$. whose wall is made up of $50 \%-50 \%$ mixture of deuterium (D) and tritium (T). Through a hole with a diameter of $10 \mu \mathrm{m}$ a beam of anti-protons is made to impinge on the wall ablating it and creating a hot dense plasma in the core. An appropriately chosen number of anti-protons and a pulse length are selected to create a plasma density of about $10^{23} \mathrm{~cm}^{-3}$ with a temperature of $20 \mathrm{keV}$ in the core to initiate the burn in the target (Cassenti, 1997). With a metal shell such as tungsten surrounding the fusion fuel, and a magnetic field of several megagaus formed to provide the needed thermal insulation, the confinement time is found to be long enough for the fusion reactions to proceed and generate a large energy content in the pellet. The large energy production is attributed to the alpha particles generated by the DT reactions whose energy is deposited in the plasma to sustain the reactions. For a plasma with an ion density $n_{i}$, the number of alpha particles per unit volume generated by the fusion reactions is given by:

$$
\mathrm{n}_{\alpha}=\frac{n_{i}^{2}}{4}\langle\sigma v\rangle(t)
$$

where $\langle\sigma v\rangle$ is the velocity averaged product of the fusion cross section $\sigma$ and relative velocity $v$ of the interacting particles. If we assume that the time $t$ is equal to the reaction time $\tau_{\mathrm{R}}=\left[n_{i}\langle\sigma v\rangle\right]^{1}$, and maintain that the alpha energy $E_{\alpha}$ of $3.5 \mathrm{MeV}$ does not get degraded in this

time period then:

$$
n_{\alpha}=\frac{n_{i}}{4}=\frac{n_{D}}{2}=\frac{n_{I}}{2},
$$

with $n_{D}$ and $n_{T}$ representing the deuterium and tritium densities respectively; then the energy content of the pellet can be primarily attributed to the alphas or:

$$
E_{T}=n_{\alpha} E_{\alpha} V
$$

where $\mathrm{V}$ is the volume of the pellet. At $20 \mathrm{keV},\langle\sigma \nu\rangle-4.2 \times 10^{-16} \mathrm{~cm}^{3} / \mathrm{sec}$. and for $\mathrm{n}_{\mathrm{i}}=10^{23}, \tau_{\mathrm{R}}$ is found to be about $2.5 \times 10^{-8}$ seconds which is about 0.250 times shorter than the plasma confinement time in MICF $\left(\sim 10^{-7} \mathrm{sec}\right.$. $)$. For a pellet of $3.5 \mathrm{gm}$. mass, the velocity with which is ejected is given by:

$$
v_{e}=\sqrt{\frac{2 E_{T}}{m_{T}}},
$$

which for the above scenario gives $\mathrm{v}_{\mathrm{e}} \cong 10^{7} \mathrm{~m} / \mathrm{sec}$. and a specific impulse $\mathrm{I}_{\mathrm{sp}} \sim 10^{6}$ seconds. 


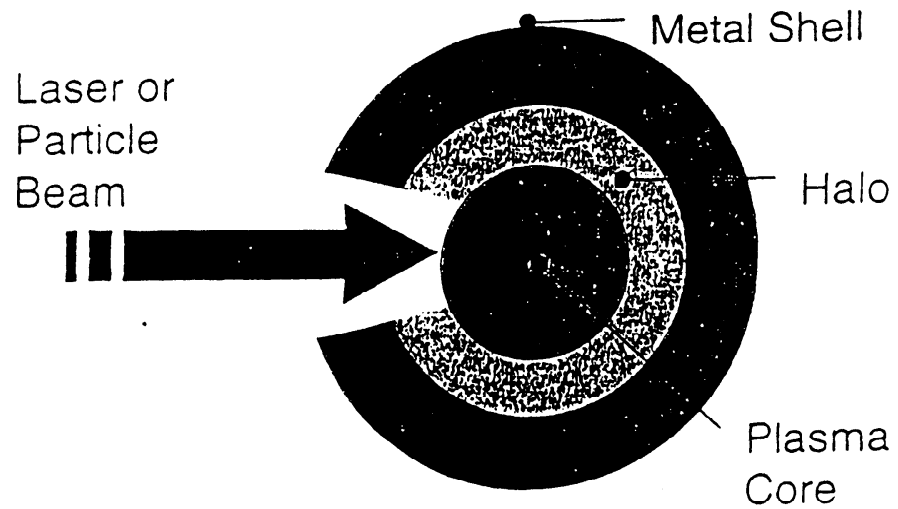

(1)

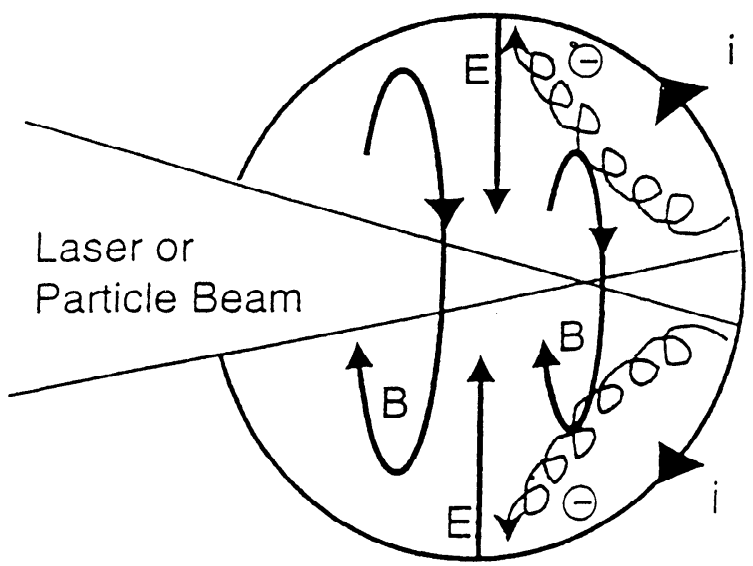

(2)

FIGURE 1. Schematic of (1) Plasma Formation and (2) Magnetic Field Formation in MICF.

The above result however may be somewhat optimistic since, as we shall see shortly, the alpha particle slows down in the plasma in a time which is much shorter than the reaction time. The slowing down process takes place as a result of interaction of the alpha particle with the electrons and ions of the plasma as represented by (Kammash, 1975).

$$
\frac{d E}{d t}=C_{1} E+C_{2} / \sqrt{E}
$$

where $\mathrm{C}_{1}$ and $\mathrm{C}_{2}$ are given by:

$$
\begin{aligned}
\mathrm{C}_{\mathrm{l}}=2 \times 10^{-12} \mathrm{n}_{\mathrm{i}}\left(\mathrm{cm}^{-3}\right) / \mathrm{T}_{\mathrm{e}}^{3 / 2}(\mathrm{keV}) \\
\mathrm{C}_{2}=9 \times 10^{-10} \mathrm{n}_{\mathrm{i}}\left(\mathrm{cm}^{-3}\right) / \mathrm{M}_{\mathrm{l}}(\mathrm{amu})
\end{aligned}
$$


When the plasma confinement time in the pellet is much longer than the reaction or slowing down times then the alpha particle density can be expressed by (Kammash, 1975)

$$
N_{\alpha}=\int n_{\alpha}(E) d E
$$

where the alpha density per unit energy $\mathrm{n}_{\alpha}(E)$ is given by:

$$
n_{\alpha}(E)=\frac{\frac{n_{i}^{2}}{4}\langle\sigma v\rangle}{d E / d t},
$$

with the denominator given by Eq. (5). Integration of Eq. (5) between the thermal energy $\mathrm{E}_{\mathrm{th}}$ of $20 \mathrm{keV}$ and the alpha birth energy of $\mathrm{E}_{0}=3.5 \mathrm{meV}$ yields the thermalization time $\tau_{\mathrm{Th}} \cong 2.32 \times 10^{-9} \mathrm{sec}$., which we observe to be much shorter than $\tau_{R}$. This simply indicates that the alphas thermalize almost instantly after birth and their energy goes into heating the plasma. $\Lambda$ quick estimate of this heating can be obtained by assuming alpha slowing down to be on the electrons only (i.e. ignoring the last term of Eq. 5). using Eqs. (9) and (5) into (8) we find that $\mathrm{N}_{\alpha}-2.44 \mathrm{x}$ $10^{21} \mathrm{~cm}^{-3}$. The change in the electron temperature $T_{\mathrm{e}}$ due to alpha heating can be written as (Kammash, 1992):

$$
\frac{3}{2} \Delta T_{e}=\frac{R E_{0}}{1+R},
$$

where $R=N_{\alpha} / n_{i}$. With the above result of $85 \mathrm{keV}$ added to the initial electron temperature of $20 \mathrm{keV}$, the final electron temperature of $105 \mathrm{keV}$ will also be the plasma temperature on the assumption that thermalization between the electrons and the ions of the plasma is also shorter then the confinement time. Using these results we find that $\mathrm{E}_{\mathrm{T}}$ in this case is $5.3 \times 10^{9}$ joules, and that in turn gives a pellet exhaust velocity of $\mathrm{v}_{\mathrm{e}}=1.74 \times 10^{6} \mathrm{~m} / \mathrm{sec}$. resulting in an $I_{s p}=1.74 \times 10^{5}$ seconds.

\section{MISSION ANAYLSIS}

We consider a robotic fly-by exploration mission to the Oort cloud which is about 10,000 AU away using the MICF propulsion system examined above. If we denote by $S_{\mathrm{f}}$ the distance to the destination, and by $t_{\mathrm{f}}$ the time it takes to reach it, then from the standard (non-relativistic) rocket equation we can write (Cassenti, 1999)

$$
\begin{aligned}
& \mathrm{t}_{f}=\frac{M_{i}-M_{f}}{F} v_{e}, \\
& s_{f}=\frac{M_{i} v_{e}^{2}}{F}\left[1-\frac{M_{f}}{M_{i}}+\frac{M_{f}}{M_{i}} \ln \left(\frac{M_{f}}{M_{i}}\right)\right], \\
& v_{f}=v_{e} \ln \left[\frac{1}{1-F_{t} / M_{i} v_{e}}\right],
\end{aligned}
$$


where $M_{I}$ is the initial mass of the vehicle, $M_{f}$ its dry mass, $F$ the thrust, and $v_{f}$ the final velocity of the vehicle when it reaches its destination assuming it started from rest. We assume that ten pellets are ejected per second; a rep rate of $\omega=10$ that is considered technologically feasible. We also take the dry mass to be $220 \mathrm{mT}$ which is consistent with a laser driven incrtial fusion propulsion system (Hyde, 1983) but without the laser driver on the assumption that the mass of an antiproton (or anti-hydrogen) source would be comparatively small to be neglected. With $\mathrm{S}_{\mathrm{f}}=10,000$ $\mathrm{AU}, \mathrm{F}=\omega m_{\mathrm{T}} v_{\mathrm{e}}$ we calculate $\mathrm{M}_{\mathrm{i}}$ from Eq. (12) and substitute it in Eq. (11) to calculate $\mathrm{t}_{\mathrm{f}}$. When these results are put in Eq. (13) we calculate $v_{f}$. These results for the mission in question are found to be:

$$
\begin{aligned}
& \mathrm{F}=6.09 \times 10^{4} \mathrm{~N}, \\
& \mathrm{t}_{\mathrm{f}}=28.53 \mathrm{Y}, \\
& \mathrm{v}_{\mathrm{f}}=7.36 \times 10^{6} \mathrm{~m} / \mathrm{s} \cong 0.025 \mathrm{c},
\end{aligned}
$$

where $\mathrm{c}$ is the speed of light.

\section{CONCLUSION}

We have shown that a novel fusion system that combines the favorable aspects of both magnetic and inertial fusions can produce very impressive propulsive parameters when used as a propulsion device. We have demonstrated that an MICF propulsion system driven by antiprotons is capable of making a fly-by mission to a 10,000 AU destination in about 28 years, well below the 50 year travel time often cited as desirable for such a mission.

\section{ACKNOWLEDGMENTS}

The work of one of us (TK) was supported by Universities Space Research Association.

\section{REFERENCES}

Cassenti, B. N., Kammash, T., and Galbraith, D. L., “Antiproton Catalyzed Fusion Propulsion For Interplanetary Missions," Journal of Propulsion and Power, 13, pp. 428-434, (1997).

Cassenti, B. N., and Kammash, T., "Antiproton Triggered Fusion Propulsion For Interstellar Missions," Space Technology and Applications International Forum (STAIF-99) CONF-990103, held 31 January - 4 February 1999, AIP Conference Proceedings No. 458, Vol. 1: 1-760; Vol. II: 761-1666; CD ROM.

Hasegawa, A., Daido, H., Fujita, M., Mima, K., Murakami, M., Nakai, S., Nishihara, K., Terai, K., and Yamanaka, C., "Magnetically Insulated Inertially Confined Fusion - MICF," Nuclear Fusion, 28, pp. 369-387, (1988).

Hyde, R. A., "A Laser Fusion Rocket For Interplanetary Propulsion," Lawrence Livermore National Laboratory Report, UCRL88857, (1983).

Hyde, R. A., "A Laser Fusion Rocket For Interplanetary Propulsion," Lawrence Livermore National Laboratory Report, UCRL88857, (1983).

Kammash, T., and Galbraith, D. L., "A Iligh Gain Fusion Reactor Based On The Magnetically Insulated Inertial Confinement Fusion (MICF) Reactor," Nuclear Fusion, 29, pp. 1079-1099, (1989).

Kammash, T., and Galbraith, D. L., "A Fusion Reactor For Space Applications," Fusion Technology, 12, pp. 11-21, (1987).

Kammash, T., "Fusion Reactor Physics, Principles and Technology," Ann Arbor Science Publishers, Ch. 3, (1975).

Kammash, T., and Galbraith, D. L., "Antimatter-Driven Fusion Propulsion System For Solar System Exploration," Journal of Propulsion and Power, 8, pp. 644-649, (1992).

Sawanakamp, S., and Kammash, T., "Estimate Of The Magnetic Field Generated In The Magnetically Insulated Fusion Reactor," Bulletin American Physical Society, 31, p. 1411, (1986). 